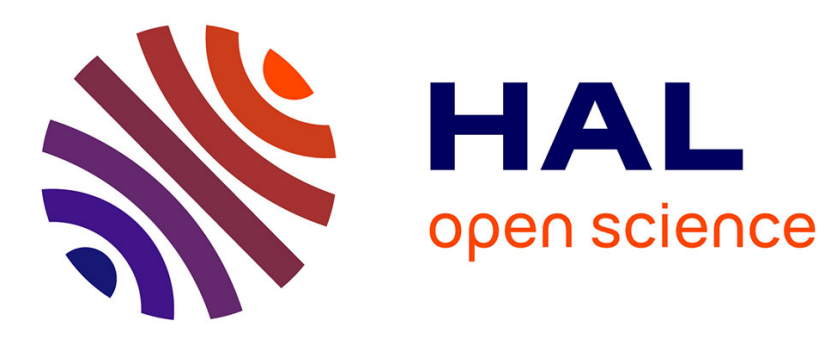

\title{
Maximum Lifetime Target Coverage in Wireless Sensor Networks
}

Nora Saadi, Ahcène Bounceur, Reinhardt Euler, Massinissa Lounis, Madani Bezoui, Moussa Kerkar, Bernard Pottier

\section{- To cite this version:}

Nora Saadi, Ahcène Bounceur, Reinhardt Euler, Massinissa Lounis, Madani Bezoui, et al.. Maximum Lifetime Target Coverage in Wireless Sensor Networks. Wireless Personal Communications, 2020, 111, pp.1525-1543. 10.1007/s11277-019-06935-5 . hal-02501797

\section{HAL Id: hal-02501797 \\ https://hal.science/hal-02501797}

Submitted on 16 Mar 2020

HAL is a multi-disciplinary open access archive for the deposit and dissemination of scientific research documents, whether they are published or not. The documents may come from teaching and research institutions in France or abroad, or from public or private research centers.
L'archive ouverte pluridisciplinaire HAL, est destinée au dépôt et à la diffusion de documents scientifiques de niveau recherche, publiés ou non, émanant des établissements d'enseignement et de recherche français ou étrangers, des laboratoires publics ou privés. 


\title{
Maximum Lifetime Target Coverage in Wireless Sensor Networks
}

\author{
Nora Saadi ${ }^{a, c}$, Ahcène Bounceur ${ }^{d}$, Reinhardt Euler ${ }^{d}$, Massinissa Lounis ${ }^{a, c}$, Madani Bezoui ${ }^{e}$, \\ Moussa Kerkar ${ }^{b, c}$, Bernard Pottier ${ }^{d}$ \\ ${ }^{a}$ LIMED Laboratory, Computer Science Department \\ ${ }^{b}$ Laboratoire de Mecanique, Materiaux et Energetique (L2ME). \\ ${ }^{c}$ Faculty of exact sciences, \\ University of A.MIRA, Bejaia, Algeria. \\ ${ }^{d}$ Lab-STICC laboratory \\ University of Western Brittany, Brest, France. \\ ${ }^{e}$ University of Boumerdes, Department of Mathematics \\ Boumerdes, Algeria. \\ Email addresse: nora1.saadi@univ-bejaia.dz
}

\begin{abstract}
The challenge in the deployment of wireless sensor networks is to ensure the coverage of targets with high energy efficiency, particularly when coverage constraint and energy constraint must be taken into account. Many algorithms have been suggested over the years to enhance network lifetime as sensor nodes are powered by batteries. These algorithms divide a set of sensor nodes into a number of subsets which can monitor all targets. The subsets are either joint or disjoint. In this paper, we propose an efficient method to compute the maximum lifetime target coverage. Our proposed method is centered around a target that is covered by a minimum number of sensor nodes. Based on this approach, we also propose an algorithm to expand the network lifetime which guarantees the monitoring of all targets by forming subsets of sensor nodes. Our obtained results are compared with the work reported in [1]. They are also compared with a method of computing the maximum lifetime target coverage (MLTC). The results obtained by simulation show that the proposed algorithm enhances the network lifetime, which illustrates the efficiency of this algorithm.
\end{abstract}

Keywords: WSN, WSN-Lifetime, Target coverage, Sensor scheduling.

\section{Introduction}

Research on wireless sensor networks (WSNs) is focusing on applications to supervise the environment, management of risks and security. A WSN is defined as a network of devices called sensor nodes, which are distributed in the space to work together using communications. Information gathered from the observed field are processed inside the network to produce internal control decisions as well as historical data stored on servers [2]. The progress in microelectronics and wireless technologies nowadays allows to manufacture tiny and cheap devices. As a result, WSNs are deployed in environments to observe physical conditions such as humidity, temperature, light, and to enable more elaborate measures for pollution, flooding or wildlife diagnostics. Energy efficiency in a WSN is an important problem due to battery of harvesting limitation. Managing the lifetime of wireless sensor networks has been bound to the notion of sensor coverage; a fundamental metric that participates in the reliability of a WSN. Coverage in wireless sensor networks is a parameter that measures for how long the sensor nodes are reliable to yield information from the phenomena of interest [3]. Target coverage is the ability of the WSN to include particular objects or zones in its coverage. For example, these may be an object or a set of objects existing, appearing or acting in an area of interest. Target coverage is a decisive property in application fields such as environment, military, medical or health care. It also improves the application quality by using optimization techniques applied to the control of sensor node activities, with effects on the energy management and the lifetimes of WSNs.

As a reference for research allowing to increase network lifetime in WSNs, a method has been proposed in [1], which allows to organize the sensor nodes into subsets that are activated 
sequentially, and in which a set of active sensor nodes is responsible for the monitoring of all the targets. To solve the problem of target coverage, the authors in [4] use a non-linear programming form to model this problem. After that, they decompose the original formulation into sub-formulations using a column generation approach and they iteratively solve them in a way that approaches the optimal solution.

To solve the target coverage problem, we propose a new offline and centralized algorithm that allows the division of a set of sensor nodes into several subsets that are activated successively. Each subset monitors all targets. To evaluate the efficiency of the proposed algorithm, we first compare the network lifetime obtained with the algorithm to the maximum lifetime target coverage (MLTC). Simulation results indicate that the network lifetime obtained by executing the algorithm converges to MLTC. We have also compared our algorithm with the maximum set cover (MSC) greedy algorithm reported in [1]. Simulation results show that our algorithm enhances the network lifetime in comparison to the algorithm presented in [1]. This paper is organized as follows: Section 2 provides a review of related work, Section 3 defines the target coverage problem and Section 4 presents the lifetime aspects. In Section 5 we present the proposed algorithm to solve the target coverage problem in WSNs, and in Section 6 simulation results are presented. The last section is devoted to the conclusion and perspectives for future work.

\section{Related work}

Maximization of target coverage is a challenging task in WSNs because the sensor nodes are powered by a battery; a reason why it is difficult to assure the monitoring of all targets for a long period. Kim et al. [5] propose an algorithm for high lifetime scheduling. To form a maximum of cover sets, the algorithm selects the sensor nodes that have the highest level of residual energy and which cover a maximum number of targets. Dong et al. [6] propose an algorithm that considers both the remaining energy of each sensor node and the number of targets that are covered by these sensor nodes to get the cover sets. In [7], Zorbas et al. present an algorithm to obtain a maximum of both joint and disjoint cover sets capable to monitor all targets. This algorithm considers the monitoring capabilities of sensor nodes by using a cost function, such as energy and sensing capability to find the best sensor nodes to include in the current cover set. Zhao et al. [8] have proposed a method that groups the sensor nodes of the network into cover sets, each of which can preserve both connectivity of the network and coverage of targets of the network among all active sensor nodes and the sink node. This problem is modelled as a maximum cover tree problem to ensure the coverage in a heterogeneous network, and it finds the optimum deployment of the sensor nodes. He et al. [9] propose a solution for the problem of target coverage using probability theory. The authors designed a heuristic that allows the organization of the sensor nodes into sensor covers, which are activated to monitor all targets and in which each cover set has a probability of failure.

The problem of holes appears when some sensor nodes are out of order of collaboration due to their failures and bad behaviour Jiang et al. [10] use directed Hamilton cycle snake-like cascading with synchronization to replace a disabled sensor node in a vacant area with a spare node. Khedr et al. [11] propose an algorithm that applied the technique divide-and-conquer to share the monitoring area into sub-regions. In each of them, a minimum number of connecting sensor nodes are selected. The authors have used Dijkstra's algorithm with a modification to find 
the shortest path between the redundant sensor nodes and the failed ones to replace the failed sensor nodes by the redundant ones. Cardei et al. [12] propose a model for scheduling the target coverage problem for WSNs with an adjustable sensing range. They propose a mathematical model, a centralized and a distributed heuristic to maximize the network lifetime. In [13], a method for scheduling the sensor is proposed, it based on integrating a learning automaton in each sensor node which helps to decide its state (sleep or active mode). Mini et al. [14] use an artificial bee colony algorithm to identify optimal deployment locations and to schedule the sensor nodes so that the network reaches a maximum lifetime. In the proposed method, only a minimum number of sensor nodes needed for favourable coverage must be activated. The other sensor nodes can be reserved for later use requirements. The aim of this method is to avoid the battery drain of all sensor nodes at a time and to extend the network lifetime. Hai Liu et al. [15] propose a method for scheduling the sensor nodes. the method based on three steps. In the first step, they compute the maximum lifetime of the network, the results being loaded into a matrix using linear programming. In the second step, the authors adapt Hall's theory by using a perfect k-matching method to divide the matrix into a sequence of schedule matrices. In the third step, they form a timetable for each sensor in the schedule matrices.

Abdelkhalek et al. [16] propose a model based on a genetic algorithm with multicriteria objective function. The solution guarantees an optimal placement of the sensor nodes. This model is implemented in a multi-agent simulator (Inform-lab). Wang et al. [17] propose a protocol called Coverage Configuration Protocol (CCP) to solve the coverage problem and connectivity in WSNs. This protocol allows the self-configuration of the network which can preserve the coverage and connectivity of the network in the goal to stand to the requirements of applications and environment. Dhawan et al. [18] propose a framework to develop heuristics solving the coverage problem. This framework is used to solve the k-coverage problem, the target coverage problem and the area coverage and within this framework local solutions are used to obtain a global one. In [19] The authors have proposed a method to produce automatically the representation of the topologies of the complex sensor networks that have several distant WSN joined with a getaway which is controlled by mobile sensors. For synchronization the authors have used concurrent process architectures and graphic accelerators. In [20] Katti proposed an energy efficient target coverage algorithm to form a joint and disjoint cover sets for monitoring targets. To preserve the energy of sensor nodes the algorithm finds the optimal path between the sink and the sensor node, and between the cover set and the sink. The algorithm saves a track of the number of targets, that are the sensor node monitor and its remaining energy in order to find the optimal path that increases the lifetime of network. Manju et al. [21] used a genetic algorithm to propose a solution heuristic to target coverage problem, that groups the sensor nodes of the network into subsets covering all targets, to form this subsets the authors select the minimum number of sensor nodes which have the maximum of remaining energy. In [22] The authors proposed a randomize algorithm to solve the target coverage problem in wireless senor networks, this algorithm allows to group the sensor nodes in not joint sets. The sensor node only belongs in one set, in this solution the active set is responsible for the supervising all targets. In [23] The authors proposed a solution for the problem of target coverage in directional sensor network, where the sensor nodes can turn around their center. In the proposed algorithm the sensor nodes are grouped in cover sets in a manner to avoid the redundant sensing direction, the obtained cover sets are joint and they are actives alternatively for monitoring all targets. 


\section{Problem statement and definitions}

The network lifetime is a fundamental parameter to take in consideration when measuring the reliability of wireless sensor network in order to ensure coverage for a long time. The target coverage problem is evoked when the sensor network is deployed to monitor targets. We define target $_{\min }$ as a particular target covered by the least number of sensor nodes and we define the network lifetime of a WSN as the time duration in which sensor nodes have the capability to monitor the phenomena of interest. The maximum lifetime target coverage (MLTC) is the sum of lifetimes of those sensor nodes which cover the target $_{\text {min }}$. We say that a sensor node monitors a target, if this target is in the sensing range of this sensor node, i.e., the distance between the sensor node and the location of the target is less than or equal to the value of the sensing range. The target coverage problem (TCP) is to find a scheduling of the sensor node activities providing a a maximum extension of the network lifetime, while ensuring the monitoring of all targets.

In this section, we use a bipartite graph to model the target coverage problem (TCP). Let us assume that we have a network with $n$ sensor nodes and $m$ targets randomly deployed. We model this network with a bipartite graph $G=(V, E)$, with $V=S \cup T$, where $S$ represents a set of sensor nodes, $T$ a set of targets and $E$ the set of edges as follows: $\{s, t\} \in E$ if and only if the sensor node $s$ detects the target $t$. We assume that each target is covered by at least one sensor node. We define the degree $d(t)$ of the target $t$ as the number of sensor nodes that detect the target $t$. All sensor nodes have the same communication range $r_{(c)}$ and the same sensing range $r_{(s)} . w \in[0,1]$ represents the lifetime of a sensor node with initial value 1 . We consider a sensor node that gathers and processes the data forwarded from the other sensor nodes as a sink node.

Example 1. Figure 1 represents a case of a network containing 6 sensor nodes and 5 targets, where a sensor node is presented by a small circle and a target is presented by a star. As represented in Figure 2, this network is modelled as a bipartite graph. The degree of a target represents the number of sensor nodes that detect this target. In our example, $d\left(t_{1}\right)=2$, for instance.

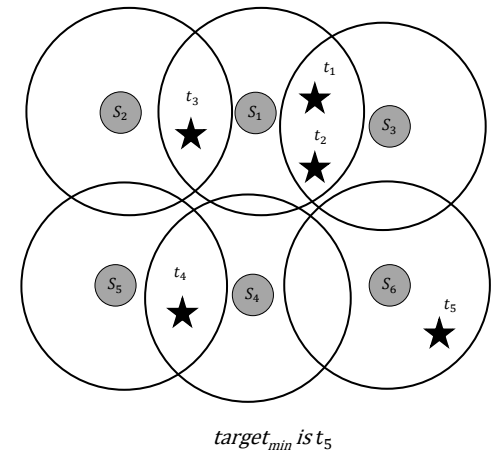

Figure 1: Example of a network with 6 sensor nodes and 5 targets

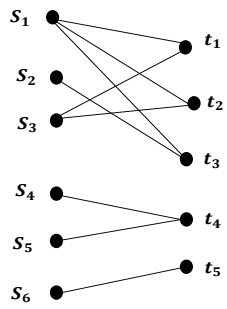

Figure 2: The representation of the network of 6 sensor nodes and 5 targets as a bipartite graph

As shown in Figures 1 and 2, a target can be detected by more than one sensor node. To avoid that sensor nodes lose their energy when supervising the same target and in order to prolong the network lifetime, we apply the maximum set cover (MSC) algorithm proposed in [1]. This algorithm allows to divide the set of sensor nodes into subsets each of them covering all targets. This algorithm also provides sensor nodes that cover a target covered by the minimum number 
of sensor nodes, to be a part of more than one subset. In Example 1, we obtain the subsets $S c_{1}=\left\{s_{1}, s_{4}, s_{6}\right\}$ and $S c_{2}=\left\{s_{2}, s_{3}, s_{5}, s_{6}\right\}$. To compute the network lifetime, we sum the lifetimes of $S c_{1}$ and $S c_{2}$ which are both equal to 0.5 , since the sensor node $s_{6}$ belongs to both $S c 1$ and $S c 2$, and its lifetime is divided by 2 . Consequently, the network lifetime equals 1 . We also observe that the only target $_{\min }$ is $t_{5}$ as it is covered by the minimum number of sensor nodes, and we deduce that at least one of the sensor nodes which cover target $_{\text {min }}$ must be turned on at the time of operating the network to guarantee the monitoring of this target $_{\text {min }}$.

\section{Example 2.}

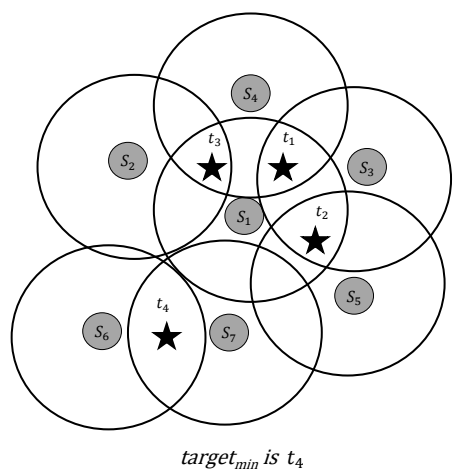

Figure 3: Example of a network with 7 sensor nodes and 4 targets

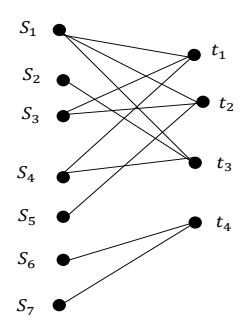

Figure 4: The representation of the network of 7 sensor nodes and 4 targets as a bipartite graph

Figure 3 represents a network with 7 sensor nodes and 4 targets and Figure 4 shows this network modelled as a bipartite graph. By applying the maximum set cover (MSC) algorithm proposed in [1], we obtain the following subsets: $S c_{1}=\left\{s_{1}, s_{6}\right\}, S c_{2}=\left\{s_{3}, s_{2}, s_{6}\right\}$ and $S c_{3}=\left\{s_{4}, s_{5}, s_{7}\right\}$. The network lifetime is the sum of the lifetimes of $S c_{1}, S c_{2}$ and $S c_{3}$. Since the lifetime of $S c_{1}$ is 0.5 , that of $S c_{2}$ is 0.5 because the sensor node $s_{6}$ belongs to both $S c_{1}$ and $S c_{2}$. and that of $S c_{3}$ is 1 , the network lifetime equals 2. In this example, target $_{\text {min }}$ is $t_{4}$ as it is covered by the minimum number of sensor nodes.

Example 3. Figure 5 represents a network with 7 sensor nodes and 6 targets and Figure 6 shows the modelling of this network as a bipartite graph, in which $t_{5}$ and $t_{6}$ are target $_{\text {min }} s$. 


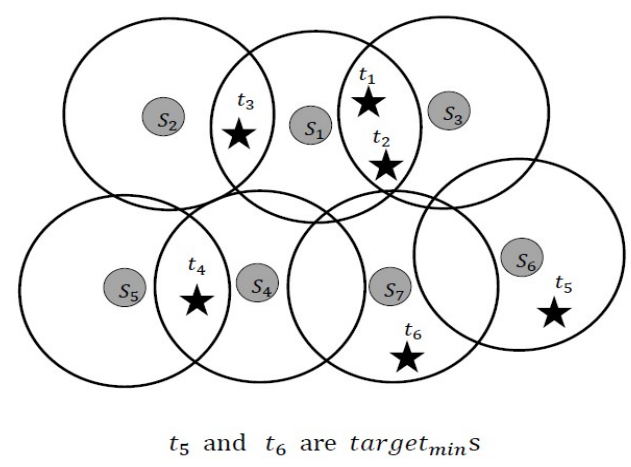

Figure 5: Example of a network with 7 sensor nodes and 6 targets

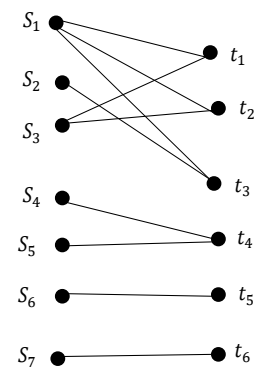

Figure 6: The representation of the network of 7 sensor nodes and 6 targets as a bipartite graph

- Case 1: If we consider that target $_{\min }$ is $t_{5}$, we obtain the following covering subsets : $S c_{1}=\left\{s_{1}, s_{4}, s_{6}, s_{7}\right\}, S c_{2}=\left\{s_{2}, s_{3}, s_{5}, s_{6}, s_{7}\right\}$. The lifetime is the sum of the lifetimes of $S c_{1}$ and $S c_{2}$. The lifetime of $S c_{1}$ is 0.5 , and that of $S c_{2}$ is 0.5 because the sensor nodes $s_{6}$ and $s_{7}$ belong to both $S c_{1}$ and $S c_{2}$, therefore the network lifetime equals 1.

- Case 2: If we consider that target $_{\min }$ is $t_{6}$, we obtain the following covering subsets : $S c_{1}=\left\{s_{1}, s_{4}, s_{6}, s_{7}\right\}, S c_{2}=\left\{s_{2}, s_{3}, s_{5}, s_{6}, s_{7}\right\}$. The network lifetime is the sum of lifetimes of $S c_{1}$ and $S c_{2}$. Since the lifetime of $S c_{1}$ is 0.5 , and that of $S c_{2}$ is 0.5 , too, because sensor nodes $s_{7}$ and $s_{6}$ belong to both $S c_{1}$ and $S c_{2}$, the network lifetime equals 1.

- Case 3: If we consider that both $t_{6}$ and $t_{7}$ are $\operatorname{target}_{m i n} s$, we obtain the same covering subsets $S c_{1}$ and $S c_{2}$, and so the network lifetime equals 1 .

Remark 1. From Examples 1 and 2, we deduce that the network lifetime depends on target $_{\text {min }}$, and the sensor nodes that cover this target must be active for the period of operating the network. Therefore, we obtain the maximum lifetime by means of the sensor nodes that monitor target $_{\text {min }}$. In the following section, we will prove that the maximum lifetime target coverage MLTC directly depends on target $_{\text {min }}$.

Remark 2. From Examples 1, 2 and 3, we deduce that if we have several target $_{\text {min }} s$ in the network, we may choose one target $_{\min }$ randomly.

\section{Maximum Lifetime target coverage in wireless sensor net- works (MLTC)}

In this section, we prove that the maximum lifetime target coverage (MLTC) is equal to the sum of the lifetimes of those sensor nodes that detect target $_{\text {min }}$. We say that the network is reliable if each target is detected by at least one sensor node. In this section, we use a mathematical model to prove that MLTC equals the sum of lifetimes of those sensor nodes that detect target $_{\text {min }}$. Let us consider a network with sensor nodes $S=\left\{s_{1}, s_{2}, \cdots, s_{n}\right\}$ and targets $T=\left\{t_{1}, t_{2}, \cdots, t_{m}\right\}$ that are deployed randomly. We assume that each target is covered by at least one sensor. Assume, that $G=(V, E)$ is such that: 


$$
\begin{aligned}
& V=S \cup T \\
& S \cap T=\emptyset \\
& |S|=n \\
& |T|=m
\end{aligned}
$$

Definition 1. We define a target detection function as follows:

$$
\operatorname{detect}(s, t)= \begin{cases}1 & \text { if the sensor s detects the target } t \\ 0 & \text { else }\end{cases}
$$

Assume, that a set of sensor nodes $S$ is decomposed into $k$ subsets, $S=\left\{S c_{1}, S c_{2}, \cdots, S c_{k}\right\}$ such that each subset guarantees the coverage of all targets.

Definition 2. We define the subset of sensor nodes that detect target $t$, as follows: $S(t)=\{s \in S, \operatorname{detect}(s, t)=1\}$.

We also let $t_{\min }$ denote the cardinality of the sensor nodes which cover target $_{\text {min }}$, i.e. $t_{\text {min }}=\mid S\left(\right.$ target $\left._{\min }\right) \mid$.

Definition 3. We define a function $g$ as follows:

$\forall i \in\{1, \ldots, k\}$

$$
g\left(S c_{i}, s\right)= \begin{cases}1 & \text { if } s \in S c_{i} \\ 0 & \text { otherwise }\end{cases}
$$

Definition 4. We define a function $f(s)$ to count the number of occurrences of a sensor in all subsets $S c_{1}, S c_{2}, \cdots, S c_{k}$ as follows:

$$
f(s)=\sum_{i=1}^{k} g\left(S c_{i}, s\right), \forall s \in S .
$$

Proposition 4.1. $\exists f_{\min }, f_{\max } \in \mathbb{N}$ such that

$$
1 \leq f_{\min } \leq f(s) \leq f_{\max } \leq k, \forall s \in S
$$

and $\exists t_{\max } \in \mathbb{N}$ such that

$$
1 \leq t_{\min } \leq|S(t)| \leq t_{\max } \leq n, \forall t \in T \text {. }
$$

Proof.

$$
\sum_{s \in S\left(\text { target }_{\text {min }}\right)} f(s) \geq k
$$

since if

$$
\sum_{s \in S\left(\text { target }_{\text {min }}\right)} f(s)<k .
$$

$\exists i \in\{1, \ldots, k\}$ such that $S c_{i} \cap S\left(\right.$ target $\left._{\min }\right)=\emptyset$, therefore there is a contradiction. 
Definition 5. The lifetime $L\left(S c_{i}\right)$ of $S c_{i}$ is defined as follows:

$$
L\left(S c_{i}\right)=\min _{s \in S c_{i}} \frac{1}{f(s)}, \forall i \in\{1, \ldots, k\}
$$

We conclude that the maximum network lifetime $L F(S)$ is given by

$$
L F(S)=\sum_{i=1}^{k} L\left(S c_{i}\right)
$$

Theorem 1. The maximum network lifetime that provides coverage of all targets is equal to the sum of the lifetimes of sensor nodes that cover target $_{\text {min }}$, i.e.

$$
L F(S)=t_{\min }
$$

Proof. 1. We suppose that:

$$
L F(S)>t_{\min }
$$

For all $i \in\{1, \ldots, k\}$, we have $L\left(S c_{i}\right) \leq 1$, which implies

$$
L F(S) \leq k .
$$

But then

$$
k>t_{\min } .
$$

From Equation (3), we deduce:

$$
\sum_{s \in S\left(\text { target }_{\min }\right)} f(s)>t_{\text {min }}
$$

which implies that $\exists s_{\alpha} \in S\left(\right.$ target $\left._{\min }\right), f\left(s_{\alpha}\right)=\alpha$, such that $\alpha>1$.

Then:

$$
\sum_{s_{\alpha} \in S\left(\text { target }_{\text {min }}\right)} f\left(s_{\alpha}\right)=t_{\text {min }}+\alpha-1
$$

From Equations (3) and (11)

$$
t_{\text {min }}+\alpha-1 \geq k
$$

Let

$k_{\alpha}=\left\{S c_{i} \in S, s_{\alpha} \in S c_{i}\right\}$

$k_{\alpha} \subset S$ and $\left|k_{\alpha}\right|=\alpha$.

$$
L F(S)=\sum_{S c_{i} \in k_{\alpha}} L\left(S c_{i}\right)+\sum_{S c_{i} \in\left(S-k_{\alpha}\right)} L\left(S c_{i}\right) .
$$




$$
\begin{aligned}
& \forall S c_{i} \in k_{\alpha}, L\left(S c_{i}\right) \leq \frac{1}{\alpha} \Rightarrow \sum_{S c_{i} \in k_{\alpha}} L\left(S c_{i}\right) \leq\left|k_{\alpha}\right| \times \frac{1}{\alpha} \\
& \Rightarrow \sum_{S c_{i} \in k_{\alpha}} f\left(S c_{i}\right) \leq 1 \\
& (13) \Rightarrow L F(S) \leq \sum_{S c_{i} \in\left(S-k_{\alpha}\right)} L\left(S c_{i}\right)+1 \\
& \left|S-k_{\alpha}\right|=k-\alpha \\
& \forall s_{j} \in\left(S-k_{\alpha}\right), L\left(S c_{j}\right) \leq 1
\end{aligned}
$$

$$
L F(S) \leq k-\alpha+1
$$

From Equations (12) and (14) we deduce that:

$L F(s) \leq t_{\min }+\alpha-1-\alpha+1$

Then

$$
L F(s) \leq t_{\min }
$$

which is a contradiction to (08).

2. We suppose that:

$$
L F(S)<t_{\text {min }}
$$

Then $\exists i \in\{1, \ldots, k\}$ such that $S c_{i} \cap S\left(\right.$ target $\left._{\min }\right)=\emptyset$.

and thus

$$
\sum_{s \in S\left(\text { target }_{\text {min }}\right)} f(s)<k
$$

But Equation (17) is in contradiction to the Equation (03), and therefore,

$$
L F(S) \geq t_{\min }
$$

From Equations (8) and (16) we deduce that $L F(S)=t_{\text {min }}$

\section{$5 \quad$ Proposed approach}

In this section, we propose an efficient algorithm for the coverage target problem; its goal is to enhance the network lifetime by organizing the sensor nodes into cover sets that are activated successively, these subsets guaranteeing the monitoring of all targets. The proposed algorithm depends on target $_{\min }$ as defined previously, and is as follows: 


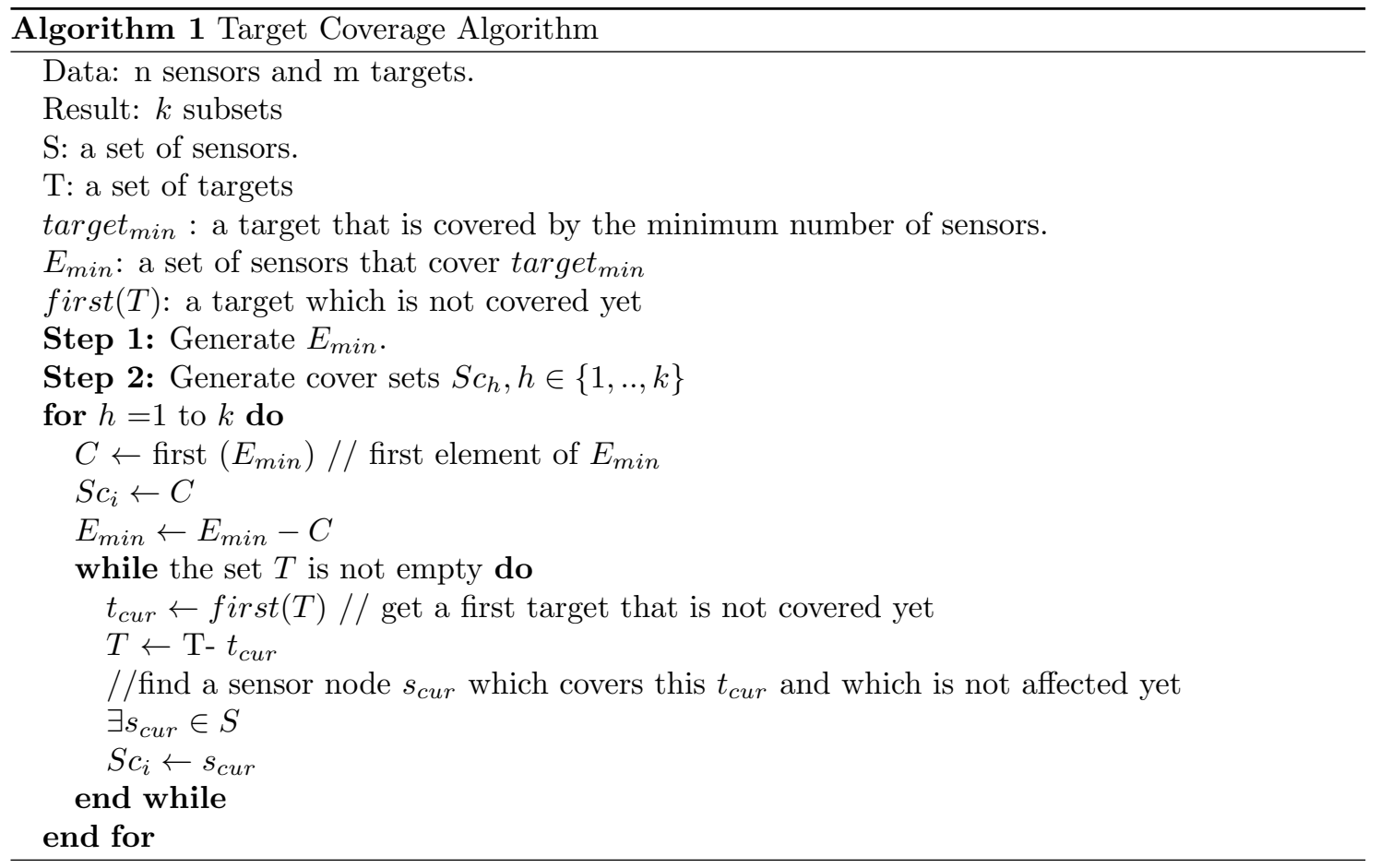

The target coverage algorithm allows the decomposition of a set of sensor nodes into $k$ subsets. In the first step, we find the target which is covered by the minimum number of sensor nodes that we have defined previously as target $_{\text {min }}$. Then we select the sensor nodes that detect target $_{\text {min }}$ and we form a subset with these sensor nodes, which we define as $E_{\min }$. After that, we delete sensor nodes of the set $E_{\min }$ and target $_{\min }$ from the sets $\mathrm{S}$ and $\mathrm{T}$, respectively. We repeat this step until all targets are covered by at least one sensor node. So, we obtain the first subset $S c_{1}$. This first procedure of selecting a subset of covering sensor nodes is performed several times to find all the possible combinations of target covering subsets.

Proposition 1. The maximum number of sensor subsets, that our algorithm may find, equals $\frac{n !}{(n-m) ! \cdot m !}$.

Proof. Indeed, we are dealing with a network where each sensor covers all targets. Therefore, there is an edge between each point of the set $S$ with each point of the set $T$. Then the problem is to select at each iteration, $m$ sensors for each of the $m$ targets. This number cannot exceed the combination of $\mathrm{m}$ out of $\mathrm{n}$ which is equal to $\left(\begin{array}{c}n \\ m\end{array}\right)=\frac{n !}{(n-m) ! . m !}$.

\section{Case study}

In this section, we explain the steps of our algorithm. Figure 7 shows a network containing 11 sensor nodes $S=\left\{s_{1}, s_{2}, \cdots, s_{11}\right\}$ and 6 targets $T=\left\{t_{1}, t_{2}, \cdots, t_{6}\right\}$. The first step of the algorithm consists in finding target $_{\min }$. In the second step, we select the subset of sensor nodes that cover this target and we form a subset that we have defined previously as $E_{\text {min }}$.

As shown in this example, the target $_{\min }$ is $t_{5}$, sensor nodes that cover this target form the subset $E_{\min }=\left\{s_{6}, s_{8}\right\}$. The next step consists in generating the cover set. For this, we select one sensor node in the subset $E_{\min }$ and we delete this sensor node from $E_{m i n}$. After that, we look for 


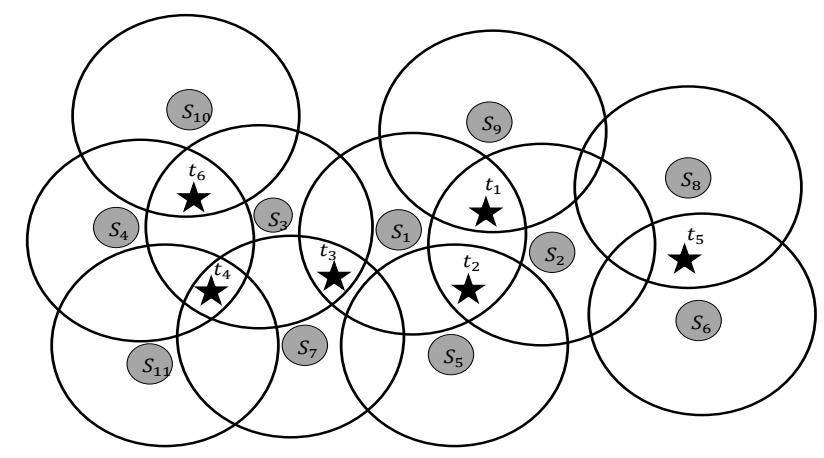

Figure 7: Example of a network of 11 sensor nodes and 6 targets

the sensor nodes that cover the other targets and we delete these sensor nodes and these targets from the sets $S$ and $T$, respectively. The subset of sensor nodes is generated until all targets are covered. We repeat this procedure of set cover until the set $E_{\min }$ becomes empty. The results of the execution of our algorithm are presented in Figure 8 that shows the set that covers all targets, i.e., $S c_{1}=\left\{s_{1}, s_{6}, s_{10}, s_{11}\right\}, S c_{2}=\left\{s_{2}, s_{3}, s_{8}\right\}$.

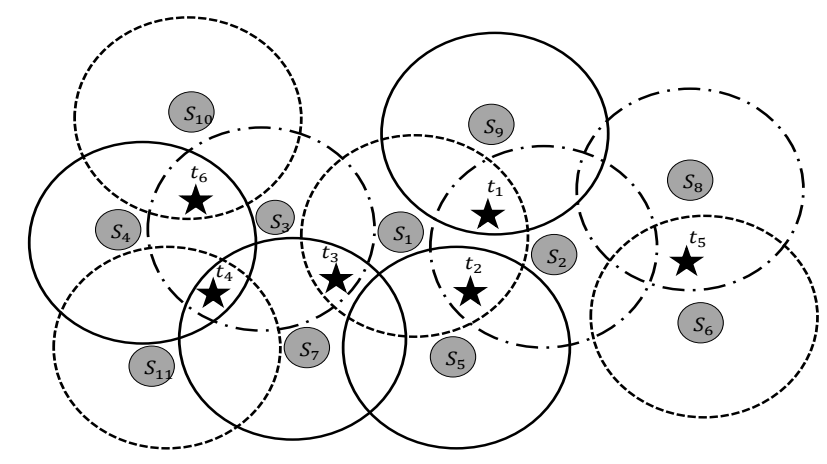

Figure 8: Subsets covering all targets obtained with our algorithm

\section{Simulation and results}

To analyse the performance of the proposed algorithm, we first compare the results of the algorithm with the maximum lifetime target coverage (MLTC) that we presented in Section 3. Next, we compare the algorithm with the greedy MSC algorithm presented in [1]. We simulate a network with target points and sensor nodes randomly positioned in an area of $500 \mathrm{~m} \times 500 \mathrm{~m}$. We assume that all sensor nodes in the network are similar and we use the same network and the same localization of targets to compare the proposed algorithm with both the maximum lifetime target coverage (MLTC) and the greedy MSC algorithm. In our simulation, we consider the following 
parameters:

- $n$, the number of sensor nodes, which should vary from 25 to 75 ,

- $m$, the number of the targets, which should vary from 5 to 15 ,

- $r$, the sensing range of each sensor node.

Subsequently, we have used the Java environment to implement these algorithms.

- In the comparison of our proposed algorithm with (MLTC), we start to use 5 randomly distributed targets and we vary the number of sensor nodes from 25 to 75 . In Figure 9, we present the network lifetime performed by our algorithm according to the number of sensor nodes in comparison to (MLTC).

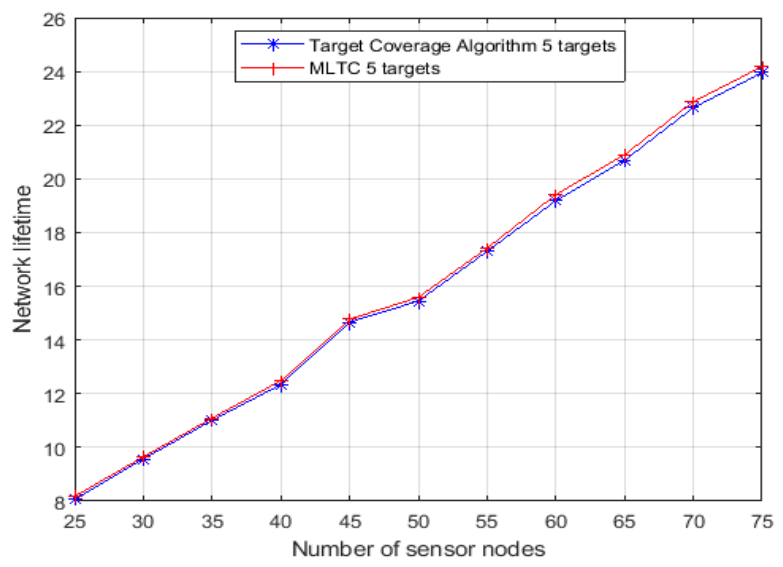

Figure 9: Comparison of the Target Coverage Algorithm with MLTC: the case of 5 targets

In the second experiment, we used 15 randomly distributed targets, and we vary the number of sensor nodes from 25 to 75 , with an increment of 5 . Figure 10 illustrates our results in comparison to (MLTC).

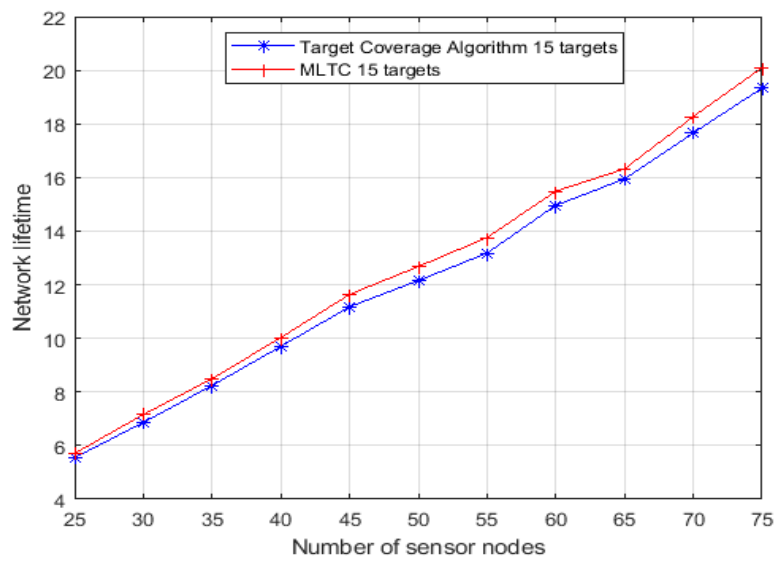

Figure 10: Comparison of the Target Coverage Algorithm with MLTC: the case of 15 targets 
The results demonstrate that the lifetime of our algorithm converges to the performance obtained with (MLTC). As illustrated in Figure 9, we have used 5 targets and 25 sensor nodes, the lifetime of the network computed by our algorithm is 8.07 and that of the (MLTC) is 8.18. As illustrated in Figure 10, we have used 15 targets and 25 sensor nodes, the lifetime of the network computed by our algorithm is 5.55 and that of (MLTC) is 5.71.

In Figure 11, we show the lifetime computed by our algorithm depending on the number of sensor nodes, in comparison to the Greedy MSC algorithm [1]. The results show that the lifetimes calculated by our algorithm are better than those obtained with the Greedy MSC algorithm. For example, in the case of 5 targets and 25 sensor nodes the lifetime of the network computed by our algorithm is 8.07 whereas the lifetime calculated by the Greedy MSC algorithm is 5.26 .

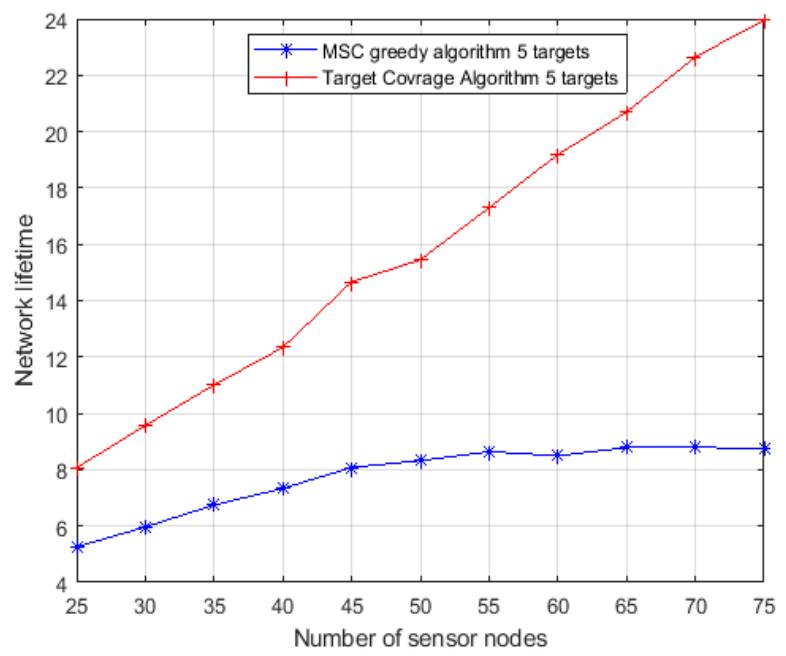

Figure 11: Comparison of the Target Coverage Algorithm with the MSC Greedy algorithm: the case of 5 targets

In the second experiment, we used 15 randomly located targets, and we varied the number of sensor nodes from 25 to 75 , with an increment of 5 . In figure 12, we represent our results in comparison MSC Greedy algorithm [1], depending on the number of sensor nodes. The results show that the lifetimes computed by our algorithm are better than the lifetime obtained by the Greedy MSC algorithm. For example, in the case of 15 targets and 25 sensor nodes the lifetime of the network computed by our algorithm is 5.55 and the lifetime computed by the Greedy MSC algorithm equals 2.9. 


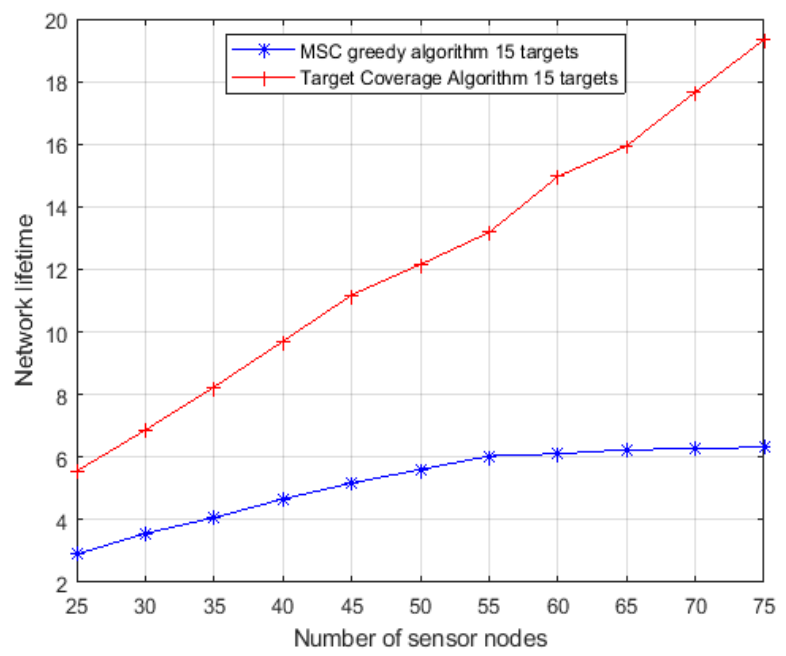

Figure 12: Comparison of the Target Coverage Algorithm with the MSC Greedy algorithm: the case of 15 targets

\section{Conclusion}

In this paper, we have presented a method to compute the maximum network lifetime of wireless sensor networks in the case of target coverage. This method is based on the target which is covered by a minimum number of sensor nodes, that is defined as target $_{\min }$. The proposed method permits to calculate the maximum lifetime target coverage MLTC which is equal to the sum of the lifetimes of the sensor nodes that cover target $_{\text {min }}$. The validity of this method has been proved mathematically in this work. Based on this method we have proposed an algorithm that solves the target coverage problem and increase the network lifetime. Its principle is to divide the entire set of sensor nodes into subsets which are activated successively for the same period. The sensor nodes of the activated subset are responsible for covering all targets and those which do not belong to the active subset are turned in sleep mode, in order to conserve their energies. The number of subsets that obtained with our algorithm is finite and does not exceed $\frac{n !}{(n-m) ! \cdot m !}$.

To prove the efficiency of the proposed algorithm, we have compared the lifetime with the maximum lifetime target coverage. Simulation results demonstrate that the lifetime obtained by our algorithm converges to MLTC, this indicates that the algorithm gives good results. We also have compared the proposed algorithm with greedy MSC algorithm [1]. The simulation results show that our algorithm extends the lifetime compared to that obtained by the algorithm presented in [1], which proves that the proposed algorithm enhances the network lifetime. Indeed, the network lifetime of our algorithm is extensively increased when we increment the number of sensor nodes, compared to that obtained by the MSC Greedy algorithm. For example in the case of 5 targets, when we vary the number of sensor nodes from 25 to 75 , the network lifetime of our algorithm increased to 15.88 and that of the MSC Greedy algorithm increased to 3.49. Whereas in the case of 15 targets, when we increment the number of sensor nodes in the same interval, the network lifetime of our algorithm is extended with a value of 13.01, and the network lifetime of Greedy's algorithm is increased with 2.65. This proves that our algorithm increases the network 
lifetime by 4 times more, compared to the MSC Greedy algorithm. So, as the number of sensor nodes augments, the difference in the network lifetimes between the two algorithms increases. After the analyses of these results, we remark that the difference of network lifetimes between our algorithm and the MSC Greedy algorithm increases from 2 to 5 we change the number of sensor nodes from 25 to 40 , and it increases by 3 times more in the interval of 40 to 75 sensor nodes.

\section{References}

[1] M.Cardei, M.T.Thai,Y.Li, W.Wu, Energy-efficient target coverage in wireless sensor networks, INFOCOM 24th Annual Joint Conference of the IEEE Computer and Communications Societies, Proceedings IEEE Vol.3, 1976-1984, 2005.

[2] P.Rawat, K.D.Singh, H.Chaouchi, J.M.Bonnin, Wireless sensor networks: a survey on recent developments and potential synergies, The Journal of supercomputing, 1-48, 2014.

[3] R.Mulligan, H.M. Ammari, Coverage in Wireless Sensor Networks: A Survey, Network Protocols and Algorithms, 27-53, 2010.

[4] Y.Gu, Y.Ji, J.Li, B.Zhao, Fundamental Results on Target Coverage Problem in Wireless Sensor Networks, Global Telecommunications Conference, 2009.

[5] H.Kim, YH.Han, S.Min, Maximum Lifetime Scheduling for Target Coverage in Wireless Sensor Networks, Proceedings of the 6th International Wireless Communications and Mobile Computing Conference, 2010.

[6] Y.Dong, J.Xu, X.Zhang, Energy-Efficient Target Coverage Algorithm for Wireless Sensor Networks, Distributed Computing Systems Workshops, 2008.

[7] D. Zorbas, D. Glynos, P. Kotzanikolaou, C. Douligeris, Solving coverage problems in wireless sensor networks using cover sets, Computer Communications Journal, Vol.8, 400-415, 2010.

[8] Zhao, Qun, M.Gurusamy, Lifetime maximization for connected target coverage in wireless sensor networks, IEEE/ACM Transactions on Networking, Vol.16, 1378-1391, 2008.

[9] J.He, S.Ji, Y.Pan, Y.Li, Reliable and Energy Efficient Target Coverage for Wireless Sensor Networks, Tsinghua Science Technology, Vol.19, 2839-2854, 2011.

[10] Z. Jiang, J. Wu, R. Kline, J. Krantz, Mobility Control for Complete Coverage in Wireless Sensor Networks, Distributed Computing Systems Workshops, 291-296, 2008.

[11] A.M. Khedr, W. Osamy, Mobility-assisted minimum connected cover in a wireless sensor network, Journal of Parallel and Distributed Computing, July 2012.

[12] M.Cardei, J.Wu, M.Lu, M.O. Pervaiz, Maximum network lifetime in wireless sensor networks with adjustable sensing ranges, IEEE WiMob, 2005.

[13] H. Mostafaei, M. Reza Meybodi, Maximizing Lifetime of Target Coverage in Wireless Sensor Networks Using Learning Automate, Wireless Personal Communications, 1461-1477, 2013.

[14] S.Mini, Siba K. Udgata, Samrat L. Sabat, Sensor deployment and scheduling for target coverage problem in wireless sensor networks, IEEE Journal, Vol.14, 636-644, 2014. 
[15] L.Hai, W.Pengjun, J.Xiaohua, Maximal lifetime scheduling for $k$ to 1 sensor target surveillance networks, Computer Networks Journal, Vol.50, 2839-2854, 2006.

[16] O. Abdelkhalek, S.Krichen, A.Guitouni, S.MitrovicMinic, A Genetic Algorithm for a MultiObjective Nodes Placement Problem in Heterogeneous Network Infrastructure for Surveillance Applications, Wireless and Mobile Networking Conference, 2011.

[17] X.Wang, G.Xing, Y.Zhang, C.Lu, R.Pless, C.Gill, Integrated coverage and connectivity configuration in wireless sensor networks, Proceedings of the 1st international conference on Embedded networked sensor systems, ACM, 28-39, 2003.

[18] A.Dhawan, Sushil K.Prasad, A distributed algorithmic framework for coverage problems in wireless sensor networks, International Journal of Parallel Emergent and Distributed Systems, 331-348, 2009.

[19] P.Y Lucas, L.N.Van, T.Phong Truong, B.Pottier, Wireless Sensor Networks and Satellite simulation, Wireless and satellite systems, Springer LNICST, pp 185-198, 2015.

[20] A.Katti, Target coverage in random wireless sensor networks using cover sets, Journal of King Saud University Computer and Information Sciences, 2019.

[21] Manju, D.Singh, S.Chand, B.Kumar, Genetic Algorithm-Based Heuristic for Solving Target Coverage Problem in Wireless Sensor Networks, Advanced Computing and Communication Technologies Springer, Singapore, 257-264, 2018.

[22] W.Luo, Z.Cai, Z.Zeng, A Simple Randomized Algorithm for Complete Target Coverage Problem in Sensor Wireless Networks, Frontier Computing Springer, Singapore, 373-378, 2016.

[23] J.Jia, C.Dong, X.He, D.Li, Y.Yu, Sensor scheduling for target coverage in directional sensor networks, International Journal of Distributed Sensor Networks, 2017. 